\title{
Existence and Global Attractivity of Positive Periodic Solutions for The Neutral Multidelay Logarithmic Population Model with Impulse
}

\author{
Zhenguo Luo, ${ }^{1,2}$ Jianhua Huang, ${ }^{1}$ Liping Luo, ${ }^{2}$ and Binxiang Dai ${ }^{3}$ \\ ${ }^{1}$ Department of Mathematics, National University of Defense Technology, Changsha 410073, China \\ ${ }^{2}$ Department of Mathematics, Hengyang Normal University, Hengyang, Hunan 421008, China \\ ${ }^{3}$ School of Mathematical Sciences and Statistics, Central South University, Changsha, Hunan 410075, China \\ Correspondence should be addressed to Zhenguo Luo; robert186@163.com
}

Received 28 January 2013; Accepted 15 April 2013

Academic Editor: Shouming Zhong

Copyright (c) 2013 Zhenguo Luo et al. This is an open access article distributed under the Creative Commons Attribution License, which permits unrestricted use, distribution, and reproduction in any medium, provided the original work is properly cited.

Sufficient and realistic conditions are established in this paper for the existence and global attractivity of a positive periodic solution to the neutral multidelay logarithmic population model with impulse by using the theory of abstract continuous theorem of $k$-set contractive operator and some inequality techniques. The results improve and generalize the known ones in $\mathrm{Li} 1999, \mathrm{Lu}$ and $\mathrm{Ge}$ 2004, Y. Luo and Z. G. Luo 2010, and Wang et al. 2009. As an application, we also give an example to illustrate the feasibility of our main results.

\section{Introduction}

In this paper, we investigate the existence and uniqueness of the positive periodic solution of the following neutral population system with multiple delays and impulse:

$$
\begin{aligned}
\frac{d N(t)}{d t}=N(t)[ & r(t)-a(t) \ln N(t) \\
& -\sum_{i=1}^{n} b_{i}(t) \ln N\left(t-\sigma_{i}(t)\right) \\
& -\sum_{j=1}^{m} c_{j}(t) \int_{-\infty}^{t} K_{j}(t-s) \ln N(s) d s \\
& \left.-\sum_{l=1}^{p} d_{l}(t) \frac{d \ln N\left(t-\tau_{l}(t)\right)}{d t}\right], \quad t \neq t_{k}, \\
N\left(t_{k}^{+}\right)= & e^{\left(1+\theta_{k}\right)} N\left(t_{k}\right), \quad k=1,2, \ldots,
\end{aligned}
$$

with the following initial conditions:

$$
\begin{array}{r}
N(\xi)=\varphi(\xi), \quad N^{\prime}(\xi)=\varphi^{\prime}(\xi), \quad \xi \in[-\tau, 0], \quad \varphi(0)>0, \\
\varphi \in C\left([-\tau, 0), R^{+}\right) \bigcap C^{1}([-\tau, 0)), R^{+},
\end{array}
$$

where $r(t), a(t), b_{i}(t), c_{j}(t), d_{l}(t), \sigma_{i}(t), \tau_{l}(t)$ are positive continuous $\omega$-periodic functions with $\sigma_{i}(t) \geq 0, \tau_{l}(t) \geq 0, t \in$ $[0, \omega], \tau=\max \left\{\sigma_{i}(t), \tau_{l}(t)\right\}, \sigma_{i}^{\prime}(t)<1, \tau_{l}^{\prime}(t)<1$, for all $i=$ $\{1,2, \ldots, n\}$, for all $l=\{1,2, \ldots, p\}$. Furthermore, $d_{l}(t) \in$ $C^{1}(R, R), \tau_{l}(t) \in C^{2}(R, R), \int_{0}^{\infty} K_{j}(s) d s=1, \int_{0}^{+\infty} s K_{j}(s) d s<$ $+\infty$, for all $j=\{1,2, \ldots, m\}$, for all $l=\{1,2, \ldots, p\}$. For the ecological justification of (1) and similar types refer to [1-7]. In recent years, Gopalsamy [1] and Kirlinger [2] had proposed the following single species logarithmic model:

$$
N^{\prime}(t)=N(t)[a-b \ln N(t)-c \ln N(t-\tau)] .
$$

In [3], Li considered the following nonautonomous single species logarithmic model:

$$
N^{\prime}(t)=N(t)[a(t)-b(t) \ln N(t)-c(t) \ln N(t-\tau(t))] .
$$


He used the continuation theorem of the coincidence degree theory to establish sufficient conditions for the existence and attractivity of positive periodic solutions of the system (4).

For more works on the periodic solution of the neutral type logistic model or the Lotka-Volterra model, see [8-12] for details. Only little scholars considered the neutral logarithmic model (see [4-7]). Li [4] had studied the following single species neutral logarithmic model:

$$
\begin{aligned}
N^{\prime}(t)=N(t)[ & r(t)-a(t) \ln N(t-\sigma) \\
& \left.-b(t)(\ln N(t-\eta))^{\prime}\right] .
\end{aligned}
$$

$\mathrm{Lu}$ and Ge [5] and Y. Luo and Z. G. Luo [6] employed an abstract continuous theorem of $k$-set contractive operator to investigate the following equation:

$$
\begin{aligned}
N^{\prime}(t)=N(t) & {\left[r(t)-\sum_{j=1}^{n} a_{j}(t) \ln N\left(t-\sigma_{j}(t)\right)\right.} \\
& \left.-\sum_{i=1}^{m} b_{i}(t)\left(\ln N\left(t-\tau_{i}(t)\right)\right)^{\prime}\right] .
\end{aligned}
$$

They established some criteria to guarantee the existence of positive periodic solutions of the system (6), respectively.

In [7], Wang et al. had investigated the existence and uniqueness of the positive periodic solution of the following neutral multispecies logarithmic population model:

$$
\begin{aligned}
N^{\prime}(t)=N(t)[ & r(t)-a(t) \ln N(t) \\
& -\sum_{j=1}^{n} b_{j}(t) \ln N\left(t-\tau_{j}(t)\right) \\
& -\sum_{j=1}^{n} c_{j}(t) \int_{-\infty}^{t} k_{j}(t-s) \ln N((s)) d s \\
& \left.-\sum_{j=1}^{n} d_{j}(t)\left(\ln N\left(t-\eta_{j}(t)\right)\right)^{\prime}\right] .
\end{aligned}
$$

By using an abstract continuous theorem of a $k$-set contractive operator, the criteria are established for the existence and global attractivity of positive periodic solutions for model (7).

On the other hand, there are some other perturbations in the real world such as fires and floods, which are not suitable to be considered continually. These perturbations bring sudden changes to the system. Systems with such sudden perturbations involving impulsive differential equations have attracted the interest of many researchers in the past twenty years [13-19], since they provide a natural description of several real processes subject to certain perturbations whose duration is negligible in comparison with the duration of the process. Such processes are often investigated in various fields of science and technology such as physics, population dynamics, ecology, biological systems, and optimal control. For details, see [20-22].
However, to this day, no scholars had done works on the existence, uniqueness, and global stability of the positive periodic solution of (1). One could easily see that the systems (5)-(7) are all special cases of the system (1). Therefore, we propose and study the system (1) in this paper.

Throughout this paper, we make the following notations.

Let $\omega>0$ be a constant,

$C_{\omega}=\{x \mid x \in C(R, R), x(t+\omega)=x(t)\}$, with the norm defined by $|x|_{0}=\max _{t \in[0, \omega]}|x(t)|$,

$C_{\omega}^{1}=\left\{x \mid x \in C^{1}(R, R), x(t+\omega)=x(t)\right\}$, with the norm defined by $\|x\|=\max _{t \in[0, \omega]}\left\{|x|_{0},\left|x^{\prime}\right|_{0}\right\}$.

Then, those spaces are both Banach spaces. We also denote that

$$
\bar{h}=\frac{1}{\omega} \int_{0}^{\omega} h(t) d t, \quad \text { for any } h \in P C_{\omega} .
$$

For the sake of generality and convenience, we always make the following fundamental assumptions:

$\left(H_{1}\right) r(t), a(t), b_{i}(t), c_{j}(t), d_{l}(t), \sigma_{i}(t), \tau_{l}(t)(i=1,2, \ldots$, $n ; j=1,2, \ldots, m ; l=1,2, \ldots, p)$ are all positive periodic continuous functions with period $\omega>0$;

$\left(H_{2}\right) 0<t_{1}<t_{2}<\cdots<t_{k}<\cdots$ are fixed impulsive points with $\lim _{k \rightarrow \infty} t_{k}=+\infty$;

$\left(H_{3}\right)\left\{\theta_{k}\right\}$ is a real sequence such that $\theta_{k}+1>0$, and $\prod_{0<t_{k}<t}\left(1+\theta_{k}\right)$ is an $\omega$-periodic function.

In the following section, some definitions and some useful lemmas are listed. In the third section, by using an abstract continuous theorem of $k$-set contractive operator and some inequality techniques, we acquired some sufficient conditions which ensure the existence and uniqueness of the positive periodic solution of the systems (1) and (2). Finally, we give an example to show our results.

\section{Preliminaries}

In order to obtain the existence and uniqueness of a periodic solution for the systems (1) and (2), we first give some definitions and lemmas.

Definition 1(see [21]). A function $N: R \rightarrow(0,+\infty)$ is said to be a positive solution of (1) and (2), if the following conditions are satisfied:

(a) $N(t)$ is absolutely continuous on each $\left(t_{k}, t_{k+1}\right)$;

(b) for each $k \in Z_{+}, N\left(t_{k}^{+}\right)$and $N\left(t_{k}^{-}\right)$exist and $N\left(t_{k}^{-}\right)=$ $N\left(t_{k}\right)$;

(c) $N(t)$ satisfies the first equation of (1) and (2) for almost everywhere (for short a.e.) in $[0, \infty] \backslash\left\{t_{k}\right\}$ and satisfies $N\left(t_{k}^{+}\right)=\left(1+\theta_{k}\right) N\left(t_{k}\right)$ for $t=t_{k}, k \in Z_{+}=$ $\{1,2, \ldots\}$.

Definition 2. The system (1) is said to be globally attractive, if there exists a positive solution $x(t)$ of (1) such that $\lim _{t \rightarrow+\infty}|x(t)-y(t)|=0$ for any other positive solution $y(t)$ of (1). 
We can easily get the following Lemma 3.

Lemma 3. The region $R=\{N(t): N(0)>0, t \geq 0\}$ is invariant with respect to (1).

Proof. In view of biological population, we obtain $N(0)>0$. By the system (1), we have

$$
\begin{aligned}
& N(t)=N(0) \\
& \times \exp \left\{\int_{0}^{t}[r(\eta)-a(\eta) \ln N(\eta)\right. \\
& -\sum_{i=1}^{n} b_{i}(\eta) \ln N\left(\eta-\sigma_{i}(\eta)\right) \\
& -\sum_{j=1}^{m} c_{j}(\eta) \int_{-\infty}^{t} K_{j}(\eta-s) \ln N(s) d s \\
& \left.\left.-\sum_{l=1}^{p} d_{l}(\eta) \frac{d \ln N\left(\eta-\tau_{l}(\eta)\right)}{d \eta}\right] d \eta\right\} \\
& >0, \quad t \in\left[0, t_{1}\right], \\
& N(t)=N\left(t_{k}\right) \\
& \times \exp \left\{\int_{t_{k}}^{t}[r(\eta)-a(\eta) \ln N(\eta)\right. \\
& -\sum_{i=1}^{n} b_{i}(\eta) \ln N\left(\eta-\sigma_{i}(\eta)\right) \\
& -\sum_{j=1}^{m} c_{j}(\eta) \int_{-\infty}^{t} K_{j}(\eta-s) \ln N(s) d s \\
& \left.\left.-\sum_{l=1}^{p} d_{l}(\eta) \frac{d \ln N\left(\eta-\tau_{l}(\eta)\right)}{d \eta}\right] d \eta\right\} \\
& >0, \quad t \in\left(t_{k}, t_{k+1}\right] \text {, } \\
& N\left(t_{k}^{+}\right)=e^{\left(1+\theta_{k}\right)} N\left(t_{k}\right)>0, \quad k \in N .
\end{aligned}
$$

Then, the solution of (1) and (2) is positive.

Under the above hypotheses $\left(H_{1}\right)-\left(H_{3}\right)$, we consider the following neutral nonimpulsive system:

$$
\begin{aligned}
\frac{d y(t)}{d t}=y(t)[ & r(t)-A(t) \ln y(t) \\
& -\sum_{i=1}^{n} B_{i}(t) \ln y\left(t-\sigma_{i}(t)\right) \\
& -\sum_{j=1}^{m} C_{j}(t) \int_{-\infty}^{t} K_{j}(t-s) \ln y(s) d s \\
& \left.-\sum_{l=1}^{p} D_{l}(t) \frac{d \ln y\left(t-\tau_{l}(t)\right)}{d t}\right],
\end{aligned}
$$

with the following initial conditions:

$$
\begin{array}{r}
y(\xi)=\varphi(\xi), \quad y^{\prime}(\xi)=\varphi^{\prime}(\xi), \quad \xi \in[-\tau, 0], \quad \varphi(0)>0, \\
\varphi \in C\left([-\tau, 0), R^{+}\right) \bigcap C^{1}([-\tau, 0)), R^{+},
\end{array}
$$

where

$$
\begin{gathered}
A(t)=a(t) \prod_{0<t_{k}<t}\left(1+\theta_{k}\right), \\
B_{i}(t)=b_{i}(t) \prod_{0<t_{k}<t-\sigma_{i}(t)}\left(1+\theta_{k}\right), \\
C_{j}(t)=c_{j}(t) \prod_{0<t_{k}<t}\left(1+\theta_{k}\right), \\
D_{l}(t)=d_{l}(t) \prod_{0<t_{k}<t-\tau_{l}(t)}\left(1+\theta_{k}\right) .
\end{gathered}
$$

By a solution $y(t)$ of (10) and (11), it means an absolutely continuous function $y(t)$ defined on $[-\tau, 0]$ that satisfies (10) a.e., for $t \geq 0$, and $y(\xi)=\varphi(\xi), y^{\prime}(\xi)=\varphi^{\prime}(\xi)$ on $[-\tau, 0]$.

The following lemmas will be used in the proofs of our results, and the proof of the lemma is similar to that of Theorem 1 in [13].

Lemma 4. Suppose that $\left(H_{1}\right)-\left(H_{4}\right)$ hold. Then,

(1) if $y(t)$ is a solution of (10) and (11) on $[-\tau,+\infty)$, then $N(t)=\prod_{0<t_{k}<t} e^{\left(1+\theta_{k}\right)} y(t)$ is a solution of (1) and (2) on $[-\tau,+\infty)$;

(2) if $N(t)$ is a solution of (1) and (2) on $[-\tau,+\infty)$, then $y(t)=\prod_{0<t_{k}<t}\left(e^{\left(1+\theta_{k}\right)}\right)^{-1} N(t)$ is a solution of $(10)$ and (11) on $[-\tau,+\infty)$.

Proof. (1) It is easy to see that $N(t)=\prod_{0<t_{k}<t} e^{\left(1+\theta_{k}\right)} y(t)$ is absolutely continuous on every interval $\left(t_{k}, t_{k+1}\right], t \neq t_{k}, k=$ $1,2, \ldots$,

$$
\begin{gathered}
N^{\prime}(t)-N(t)[r(t)-a(t) \ln N(t) \\
\quad-\sum_{i=1}^{n} b_{i}(t) \ln N\left(t-\sigma_{i}(t)\right) \\
-\sum_{j=1}^{m} c_{j}(t) \int_{-\infty}^{t} K_{j}(t-s) \ln N(s) d s \\
\left.\quad-\sum_{l=1}^{p} d_{l}(t) \frac{d N\left(t-\tau_{l}(t)\right)}{d t}\right] \\
=\prod_{0<t_{k}<t} e^{\left(1+\theta_{k}\right)} y^{\prime}(t) \\
\quad-\prod_{0<t_{k}<t} e^{\left(1+\theta_{k}\right)} y(t)
\end{gathered}
$$




$$
\begin{aligned}
& \times\left[r(t)-a(t) \prod_{0<t_{k}<t}\left(1+\theta_{k}\right) \ln y(t)\right. \\
& -\sum_{i=1}^{n} b_{i}(t) \prod_{0<t_{k}<t-\sigma_{i}(t)}\left(1+\theta_{k}\right) \\
& \times \ln y\left(t-\sigma_{i}(t)\right) \\
& -\sum_{j=1}^{m} c_{j}(t) \int_{-\infty}^{t} K_{j}(t-s) \\
& \times \prod_{0<t_{k}<t}\left(1+\theta_{k}\right) \ln y(s) d s \\
& -\sum_{l=1}^{p} d_{l}(t) \prod_{0<t_{k}<t-\tau_{l}(t)}\left(1+\theta_{k}\right) \\
& \left.\times \frac{d y\left(t-\tau_{l}(t)\right)}{d t}\right] \\
& =\prod_{0<t_{k}<t} e^{\left(1+\theta_{k}\right)} \\
& \times\left\{y^{\prime}(t)-y(t)\right. \\
& \times[r(t)-A(t) \ln y(t) \\
& -\sum_{i=1}^{n} B_{i}(t) \ln y\left(t-\sigma_{i}(t)\right) \\
& -\sum_{j=1}^{m} C_{j}(t) \int_{-\infty}^{t} K_{j}(t-s) \ln y(s) d s \\
& \left.\left.-\sum_{j=1}^{n} D_{l}(t) \frac{d y\left(t-\tau_{l}(t)\right)}{d t}\right]\right\}=0 .
\end{aligned}
$$

On the other hand, for any $t=t_{k}, k=1,2, \ldots$,

$$
\begin{aligned}
N\left(t_{k}^{+}\right) & =\lim _{t \rightarrow t_{k}^{+}} \prod_{0<t_{j}<t} e^{\left(1+\theta_{k}\right)} y(t) \\
& =\prod_{0<t_{j} \leq t_{k}} e^{\left(1+\theta_{k}\right)} y\left(t_{k}\right), \\
N\left(t_{k}\right) & =\prod_{0<t_{j}<t_{k}} e^{\left(1+\theta_{k}\right)} y\left(t_{k}\right),
\end{aligned}
$$

thus,

$$
N\left(t_{k}^{+}\right)=e^{\left(1+\theta_{k}\right)} N\left(t_{k}\right), \quad k=1,2, \ldots
$$

It follows from (13)-(15) that $N_{i}(t)$ is a solution of (1) and (2).
(2) Since $N(t)=\prod_{0<t_{k}<t} e^{\left(1+\theta_{k}\right)} y(t)$ is absolutely continuous on every interval $\left(t_{k}, t_{k+1}\right], t \neq t_{k}, k=1,2, \ldots$, and in view of (15), it follows that for any $k=1,2, \ldots$,

$$
\begin{aligned}
y\left(t_{k}^{+}\right) & =\prod_{0<t_{j} \leq t_{k}}\left(e^{\left(1+\theta_{k}\right)}\right)^{-1} N\left(t_{k}^{+}\right) \\
& =\prod_{0<t_{j}<t_{k}}\left(e^{\left(1+\theta_{k}\right)}\right)^{-1} N\left(t_{k}\right)=y\left(t_{k}\right), \\
y\left(t_{k}^{-}\right) & =\prod_{0<t_{j}<t_{k}}\left(e^{\left(1+\theta_{k}\right)}\right)^{-1} N\left(t_{k}^{-}\right) \\
& =\prod_{0<t_{j} \leq t_{k}^{-}}\left(e^{\left(1+\theta_{k}\right)}\right)^{-1} N\left(t_{k}^{-}\right)=y\left(t_{k}\right),
\end{aligned}
$$

which implies that $y(t)$ is continuous on $[-\tau,+\infty)$. It is easy to prove that $y(t)$ is absolutely continuous on $[-\tau,+\infty)$. Similar to the proof of (1), one can check that $y(t)=$ $\prod_{0<t_{k}<t}\left(e^{\left(1+\theta_{k}\right)}\right)^{-1} N(t)$ are solutions of (10) and (11) on $[-\tau,+\infty)$. The proof of Lemma 3 is completed.

Here, we take the transformation $y(t)=e^{x(t)}$; then, (10) can be rewritten in the following form:

$$
\begin{aligned}
\frac{d x(t)}{d t}= & r(t)-A(t) x(t) \\
& -\sum_{i=1}^{n} B_{i}(t) x\left(t-\sigma_{i}(t)\right) \\
& -\sum_{j=1}^{m} C_{j}(t) \int_{-\infty}^{t} K_{j}(t-s) x(s) d s \\
& -\sum_{l=1}^{p} D_{0 l}(t) x^{\prime}\left(t-\tau_{l}(t)\right),
\end{aligned}
$$

where $D_{0 l}(t)=D_{l}(t)\left(1-\tau_{l}^{\prime}(t)\right), l=1,2, \ldots, p$. Therefore, we only discuss the existence and uniqueness of a periodic solution for (17).

Definition 5 (see [23]). Let $U$ be a bounded subset in $X$. Define that

$\alpha_{X}(U)$

$$
\begin{aligned}
& =\inf \{\delta>0 \text { : there is a finite number of subsets } \\
& \left.\quad U_{i} \subset U \text { such that } U=\bigcup_{i} U_{i} \text { and } \operatorname{diam}\left(U_{i}\right) \leq \delta\right\},
\end{aligned}
$$

where $\operatorname{diam}\left(U_{i}\right)$ denotes the diameter of the set $U_{i}$, obviously, $0 \leq \alpha_{X}(U)<\infty$. So, $\alpha_{X}(U)$ is called the (Kuratowski) measure of noncompactness of $X$. 
Definition 6 (see [23]). Let $X, Y$ be two Banach spaces and $D \subset X$; a continuous and bounded map $T: D \rightarrow Y$ is called $k$-set contractive if for any bounded set $U \subset D$ one has

$$
\alpha_{Y}(T(U)) \leq k \alpha_{X}(U)
$$

$T$ is called strict-set-contractive if it is $k$-set contractive for some $0 \leq k<1$.

For a Fredholm operator $L: X \rightarrow Y$ with index zero, according to $[9,24]$, we define

$$
\begin{array}{r}
l(L)=\sup \left\{r \alpha_{X}(U) \leq \alpha_{Y}(L(U))\right. \\
\text { for any bounded set } U \subset X\} .
\end{array}
$$

Lemma 7 (see $[9,24]$ ). Let $L: X \rightarrow Y$ be a Fredholm operator with zero index and $a \in Y$ be a fixed point. Suppose that $N: \Omega \rightarrow Y$ is called a $k$-set contractive with $k<l(L)$, where $\Omega \subset X$ is bounded, open, and symmetric about $0 \in \Omega$. Further, one also assumes that

(1) $L x \neq \lambda N x+\lambda a$, for $x \in \partial \Omega, \lambda \in(0,1)$;

(2) $[Q N(x)+Q a, x][Q N(-x)+Q a, x]<0$, for $x \in$ $\operatorname{Ker} L \bigcap \partial \Omega$;

where $[\cdot, \cdot]$ is a bilinear form on $Y \times X$ and $Q$ is the projection of $Y$ onto Coker $(L)$, where Coker $(L)$ is the cokernel of the operator $L$. Then, there is an $x \in \bar{\Omega}$ such that $L x-N x=a$. $C_{\omega}$,

In order to use Lemma 7 to study (17), we set $X=C_{\omega}^{1}, Y=$

$$
L: C_{\omega}^{1} \longrightarrow C_{\omega}, \quad L x=\frac{d x}{d t}
$$

and $N: C_{\omega}^{1} \rightarrow C_{\omega}$ defined by

$$
\begin{aligned}
N x= & -A(t)(t) x(t)-\sum_{i=1}^{n} B_{i}(t) x\left(t-\sigma_{i}(t)\right) \\
& -\sum_{j=1}^{m} C_{j}(t) \int_{-\infty}^{t} K_{j}(t-s) x(s) d s \\
& -\sum_{l=1}^{p} D_{0 l}(t) x^{\prime}\left(t-\tau_{l}(t)\right) .
\end{aligned}
$$

It is easy to see from [25] that $L$ is a Fredholm operator with index zero. Thus, (17) has a positive $\omega$-periodic solution if and only if $L x=N x+r$ has a solution $x \in C_{\omega}^{1}$, where $r=r(t)$.

Lemma 8 (see [25]). The differential operator $L$ is a Fredholm operator with index zero and satisfies $l(L) \geq 1$.

Lemma 9. Let $r_{0}, r_{1}$ be two positive constants and $\Omega=\left\{x_{i} \in\right.$ $\left.C_{\omega}^{1},\left|x_{i}\right|_{o}<r_{0},\left|x_{i}^{\prime}\right|_{o}<r_{1}\right\}$. If $k=\sum_{l=1}^{p}\left|D_{0 l}\right|_{0}$, then $N: \Omega \rightarrow$ $C_{\omega}^{1}$ is a $k$-set contractive map.

As Lemma 9 can be proved in the same way as in the proof of Lemma 2.4 in [7], we omit it here.
Lemma 10 (see $[7,9]$ ). Suppose that $\tau \in C_{\omega}^{1}$ and $\tau^{\prime}(t)<1$, $t \in[0, \omega]$, then the function $t-\tau(t)$ has a unique inverse $\mu(t)$ satisfying $\mu \in C(R, R)$ with $\mu(a+\omega)=\mu(a)+\omega$ for all $a \in R$, and if $g \in P C_{\omega}, \tau^{\prime}(t)<1, t \in[0, \omega]$, then $g(\mu(t)) \in P C_{\omega}$.

Lemma 11 (see $[26,27])$. Suppose that $x(t)$ is a differently continuous $\omega$-periodic function on $R$ with $(\omega>0)$, then to any $t_{*} \in R, \max _{t_{*} \leq t \leq t_{*}+\omega}|x(t)| \leq\left|x\left(t_{*}\right)\right|+(1 / 2) \int_{0}^{\omega}\left|x^{\prime}(t)\right| d t$.

\section{Main Theorem}

Since $\sigma_{i}^{\prime}(t)<1, \tau_{l}^{\prime}(t)<1, t \in[0, \omega]$, we see that $\sigma_{i}(t), \tau_{l}(t)$ all have its inverse function. Throughout the following part, we set $\zeta_{i}(t), \xi_{l}(t)$ that represent the inverse function of $t-\sigma_{i}(t)$, $t-\tau_{l}(t)$, respectively. We also denote

$$
\begin{aligned}
\Gamma(t):= & A(t)+\sum_{i=1}^{n} \frac{B_{i}\left(\zeta_{i}(t)\right)}{1-\sigma_{i}^{\prime}\left(\zeta_{i}(t)\right)} \\
& +\sum_{j=1}^{m} C_{j}(t)-\sum_{l=1}^{p} \frac{D_{l}^{\prime}\left(\xi_{l}(t)\right)}{1-\tau_{l}^{\prime}\left(\xi_{l}(t)\right)} .
\end{aligned}
$$

Remark 12. From Lemma 9, we get that $\zeta_{i}(\omega)=\zeta_{i}(0)+$ $\omega, \xi_{l}(\omega)=\xi_{l}(0)+\omega, i=1,2, \ldots, n ; l=1,2, \ldots, p$; then,

$$
\begin{aligned}
& \int_{0}^{\omega} \frac{B_{i}\left(\zeta_{i}(s)\right)}{1-\sigma_{i}^{\prime}\left(\zeta_{i}(s)\right)} d s \\
& \quad=\int_{\zeta_{i}(0)}^{\zeta_{i}(\omega)} \frac{B_{i}(t)\left(1-\sigma_{i}^{\prime}(t)\right)}{1-\sigma_{i}^{\prime}(t)} d t \\
& \quad=\int_{\zeta_{i}(0)}^{\zeta_{i}(0)+\omega} B_{i}(t) d t=\overline{B_{i}} \omega, \quad i=1,2, \ldots, n .
\end{aligned}
$$

Similarly,

$$
\begin{aligned}
\int_{0}^{\omega} & \frac{D_{l}^{\prime}\left(\xi_{l}(s)\right)}{1-\tau_{l}^{\prime}\left(\xi_{l}(s)\right)} d s \\
\quad & =\int_{\xi_{l}(0)}^{\xi_{l}(\omega)} \frac{D_{l}^{\prime}(t)\left(1-\tau_{l}^{\prime}(t)\right)}{1-\tau_{l}^{\prime}(t)} d t \\
& =\int_{\xi_{l}(0)}^{\xi_{l}(0)+\omega} D_{l}^{\prime}(t) d t=0, \quad l=1,2, \ldots, p .
\end{aligned}
$$

Thus,

$$
\bar{\Gamma} \omega=\int_{0}^{\omega} \Gamma(t) d t=\left(\bar{A}+\sum_{i=1}^{n} \overline{B_{i}}+\sum_{j=1}^{m} \overline{C_{j}}\right) \omega .
$$

Theorem 13. In addition to $\left(H_{1}\right)-\left(H_{3}\right)$, suppose that the following conditions hold:

$\left(H_{4}\right)$ there exists a constant $\eta>0$ such that $|\Gamma(t)| \geq \eta$, for all $t \in[0, \omega]$, where $\Gamma(t)$ is defined by $(23)$;

$\left(H_{5}\right)$ if $(1 / 2)\left(|A|_{0} \omega+\sum_{i=1}^{n}\left|B_{i}\right|_{0} \omega+\sum_{j=1}^{m}\left|C_{j}\right|_{0} \omega^{1 / 2}\right)+$ $\sum_{l=1}^{p}\left|1-\tau_{l}^{\prime}\right|_{0}^{1 / 2}\left|D_{l}\right|_{0}<1$, and $\sum_{l=1}^{p}\left|1-\tau_{l}^{\prime}\right|_{0}\left|D_{l}\right|_{0}<1$.

Then, (1) has at least one positive $\omega$-periodic solution. 
Proof. Let $u(t)$ be an arbitrary $\omega$-periodic solution of the operator equation as follows:

$$
L u=\lambda N u+\lambda r, \quad \lambda \in(0,1)
$$

where $L, N$ defined by (21) and (22), respectively. Then, $u(t)$ satisfies the following operator equation:

$$
\begin{aligned}
\frac{d u(t)}{d t}=\lambda[ & r(t)-A(t) u(t) \\
& -\sum_{i=1}^{n} B_{i}(t) u\left(t-\sigma_{i}(t)\right) \\
& -\sum_{j=1}^{m} C_{j}(t) \int_{-\infty}^{t} K_{j}(t-s) u(s) d s \\
& \left.-\sum_{l=1}^{p} D_{0 l}(t) u^{\prime}\left(t-\tau_{l}(t)\right)\right] .
\end{aligned}
$$

Integrating both sides of $(28)$ over $[0, \omega]$, we have

$$
\begin{aligned}
\bar{r} \omega= & \int_{0}^{\omega}[A(t) u(t) \\
& +\sum_{i=1}^{n} B_{i}(t) u\left(t-\sigma_{i}(t)\right) \\
& +\sum_{j=1}^{m} C_{j}(t) \int_{-\infty}^{t} K_{j}(t-s) u(s) d s \\
= & \left.+\sum_{0}^{\omega} D_{l=1}^{p} D_{0 l}(t) u^{\prime}\left(t-\tau_{l}(t)\right)\right] d t \\
& +\int_{0}^{\omega} \sum_{i=1}^{n} B_{i}(t) u\left(t-\sigma_{i}(t)\right) d t \\
& +\int_{0}^{\omega} \sum_{j=1}^{m} C_{j}(t) \int_{-\infty}^{t} K_{j}(t-s) u(s) d s d t \\
& -\int_{0}^{\omega} \sum_{l=1}^{p} D_{l}^{\prime}(t) u\left(t-\tau_{l}(t)\right) d t .
\end{aligned}
$$

Let $t-\sigma_{i}(t)=s$, then $t=\zeta_{i}(s)$ and

$$
\begin{aligned}
\int_{0}^{\omega} B_{i}(t) u\left(t-\sigma_{i}(t)\right) d t \\
\quad=\int_{-\sigma_{i}(0)}^{\omega-\sigma_{i}(\omega)} \frac{B_{i}\left(\zeta_{i}(s)\right)}{1-\sigma_{i}^{\prime}\left(\zeta_{i}(s)\right)} u(s) d s
\end{aligned}
$$

By Lemma 10, we have

$$
\begin{gathered}
\int_{0}^{\omega} B_{i}(t) u\left(t-\sigma_{i}(t)\right) d t \\
\quad=\int_{0}^{\omega} \frac{B_{i}\left(\zeta_{i}(s)\right)}{1-\sigma_{i}^{\prime}\left(\zeta_{i}(s)\right)} u(s) d s .
\end{gathered}
$$

Similarly, we have

$$
\begin{aligned}
& \int_{0}^{\omega} D_{l}^{\prime}(t) u_{i}\left(t-\tau_{l}(t)\right) d t \\
& \quad=\int_{0}^{\omega} \frac{D_{l}^{\prime}\left(\xi_{l}(s)\right)}{1-\tau_{l}^{\prime}\left(\xi_{l}(s)\right)} u(s) d s .
\end{aligned}
$$

Substituting (31) and (32) into (29), we have

$$
\int_{0}^{\omega} \Gamma(s) u(s) d s=\bar{r} \omega .
$$

Considering assumption $\left(H_{4}\right)$, we know that $|\Gamma(t)| \geq \eta>0$, and then, it follows from the integro mean value theorem that there exists a $\alpha \in[0, \omega]$ satisfying

$$
|u(\alpha)|=\frac{\overline{r_{i}}}{|\Gamma(\alpha)|} \leq \frac{\overline{r_{i}}}{\eta} .
$$

By Lemma 11, we can get that

$$
|u|_{0} \leq \frac{\overline{r_{i}}}{\eta}+\frac{1}{2} \int_{0}^{\omega}\left|u^{\prime}(t)\right| d t .
$$

Multiplying both sides of (28) by $u^{\prime}(t)$ and integrating them over $[0, \omega]$, we have

$$
\begin{aligned}
& \int_{0}^{\omega}\left|u^{\prime}(t)\right|^{2} d t \\
& =\lambda \mid \int_{0}^{\omega} r(t) u^{\prime}(t) d t-\int_{0}^{\omega} A(t) u(t) u^{\prime}(t) d t \\
& \quad-\int_{0}^{\omega} \sum_{i=1}^{n} B_{i}(t) u\left(t-\sigma_{i}(t)\right) u^{\prime}(t) d t \\
& \quad-\int_{0}^{\omega} \sum_{j=1}^{m} C_{j}(t) \int_{-\infty}^{t} K_{j}(t-s) u(s) u^{\prime}(t) d s d t \\
& \quad-\int_{0}^{\omega} \sum_{l=1}^{p} D_{0 l}(t) u^{\prime}\left(t-\tau_{l}(t)\right) u^{\prime}(t) d t \mid \\
& +\quad \int_{0}^{\omega} \sum_{l=1}^{p}\left|D_{0}+(t) u^{\prime}\left(t-\tau_{l}(t)\right) u^{\prime}(t)\right| d t . \\
& +\int_{0}^{\omega} \sum_{j=1}^{m}\left|C_{j}(t) \int_{-\infty}^{t} K_{j}(t-s) u(s) d s u^{\prime}(t)\right| d t
\end{aligned}
$$


International Journal of Engineering Mathematics

7

By using the Cauchy-Schwarz inequality, we get that

$$
\begin{aligned}
& \int_{0}^{\omega}\left|u^{\prime}(t)\right|^{2} d t \\
& \leq\left[|r|_{0}+\left(|A|_{0}+\sum_{i=1}^{n}\left|B_{i}\right|_{0}\right)|u|_{0}\right] \\
& \times\left(\int_{0}^{\omega}\left|u^{\prime}(t)\right|^{2} d t\right)^{1 / 2} \omega^{1 / 2} \\
& +\left(\sum_{j=1}^{m}\left|C_{j}(t) \int_{-\infty}^{t} K_{j}(t-s) u(s) d s\right|^{2} d t\right)^{1 / 2} \\
& \times\left(\int_{0}^{\omega}\left|u^{\prime}(t)\right|^{2} d t\right)^{1 / 2} \\
& +\sum_{l=1}^{p}\left(\int_{0}^{\omega}\left|D_{0 l}(t) u^{\prime}\left(t-\tau_{l}(t)\right)\right|^{2} d t\right)^{1 / 2} \\
& \times\left(\int_{0}^{\omega}\left|u^{\prime}(t)\right|^{2} d t\right)^{1 / 2} \\
& =\left[|r|_{0} \omega^{1 / 2}+\left(|A|_{0} \omega^{1 / 2}+\sum_{i=1}^{n}\left|B_{i}\right|_{0} \omega^{1 / 2}+\sum_{j=1}^{m}\left|C_{j}\right|_{0}\right)|u|_{0}\right] \\
& \times\left(\int_{0}^{\omega}\left|u^{\prime}(t)\right|^{2} d t\right)^{1 / 2} \\
& +\sum_{l=1}^{p}\left(\int_{0}^{\omega}\left|D_{0 l}(t) u^{\prime}\left(t-\tau_{l}(t)\right)\right|^{2} d t\right)^{1 / 2} \\
& \times\left(\int_{0}^{\omega}\left|u^{\prime}(t)\right|^{2} d t\right)^{1 / 2} .
\end{aligned}
$$

Meanwhile, we see that

$$
\begin{aligned}
& \left(\int_{0}^{\omega}\left|D_{0 l}(t) u^{\prime}\left(t-\tau_{l}(t)\right)\right|^{2} d t\right)^{1 / 2} \\
& \quad=\left(\int_{0}^{\omega} \frac{1}{1-\tau_{l}^{\prime}\left(\xi_{l}(t)\right)}\left|D_{0 l}\left(\xi_{l}(t)\right) u^{\prime}(t)\right|^{2} d t\right)^{1 / 2} \\
& \quad=\left(\int_{0}^{\omega}\left(1-\tau_{l}^{\prime}\left(\xi_{l}(t)\right)\right)\left|D_{l}\left(\xi_{l}(t)\right) u^{\prime}(t)\right|^{2} d t\right)^{1 / 2} \\
& \quad \leq\left|1-\tau_{l}^{\prime}\right|_{0}^{1 / 2}\left|D_{l}\right|_{0}\left(\int_{0}^{\omega}\left|u^{\prime}(t)\right|^{2} d t\right) .
\end{aligned}
$$

From $\left(H_{5}\right)$, we get that $(1 / 2)\left(|A|_{0} \omega+\sum_{i=1}^{n}\left|B_{i}\right|_{0} \omega+\right.$ $\left.\sum_{j=1}^{m}\left|C_{j}\right|_{0} \omega^{1 / 2}\right)+\sum_{l=1}^{p}\left|1-\tau_{l}^{\prime}\right|_{0}^{1 / 2}\left|D_{l}\right|_{0}<1$. Then, there exists a constant $N>0$ such that

$$
\left(\int_{0}^{\omega}\left|u_{i}^{\prime}(t)\right|^{2} d t\right)^{1 / 2} \leq N .
$$


From (35) and the Hölder inequality, we obtain that

$$
\begin{aligned}
|u|_{0} & \leq \frac{\bar{r}}{\eta}+\frac{1}{2} \int_{0}^{\omega}\left|u^{\prime}(t)\right| d t \\
& \leq \frac{\bar{r}}{\eta}+\frac{\omega^{1 / 2}}{2}\left(\int_{0}^{\omega}\left|u^{\prime}(t)\right|^{2} d t\right)^{1 / 2} \leq \frac{\bar{r}}{\eta}+\frac{\omega^{1 / 2}}{2} N:=N_{1} .
\end{aligned}
$$

Again from (28), we get that

$$
\begin{aligned}
\left|u^{\prime}\right|_{0} \leq & |r|_{0}+\left(|A|_{0}+\sum_{i=1}^{n}\left|B_{i}\right|_{0}+\sum_{j=1}^{m}\left|C_{j}\right|_{0}\right)|u|_{0} \\
& +\sum_{l=1}^{p}\left|D_{0 l}\right|_{0}\left|u^{\prime}\right|_{0} .
\end{aligned}
$$

From condition $\sum_{l=1}^{p}\left|D_{0 l}\right|_{0} \leq \sum_{l=1}^{p}\left|1-\tau_{l}^{\prime}\right|_{0}\left|D_{l}\right|_{0}<1$, it is easy to see that

$$
\left|u^{\prime}\right|_{0} \leq \frac{|r|_{0}+\left(|A|_{0}+\sum_{i=1}^{n}\left|B_{i}\right|_{0}+\sum_{j=1}^{m}\left|C_{j}\right|_{0}\right) N_{1}}{1-\sum_{l=1}^{p}\left|D_{0 l}\right|_{0}}:=N_{2} \text {. }
$$

Now, we take $N_{3}>\max \left\{N_{1}, N_{2},\left|\bar{r} /\left(\bar{A}+\sum_{i=1}^{n} \overline{B_{i}}+\sum_{j=1}^{m} \overline{C_{j}}\right)\right|\right\}$ and $\Omega=\left\{u \mid u \in C_{\omega}^{1},\|u\|<N_{3}\right\}$. Then, $k=\sum_{l=1}^{p}\left|D_{0 l}\right|_{0}<1 \leq$ $l(L)$. So by (42) and (44), we can find that all the conditions of Lemma 4 except (2) hold. In what follows, we will prove that condition (2) of Lemma 4 is also satisfied. In order to do this, we defined a bounded bilinear form on $C_{\omega}, C_{\omega}^{!}$by $[\cdot, \cdot]$ as the following $[y, x]=\int_{0}^{\omega} y(t) x(t) d t$. Also, we defined $Q: y \rightarrow$ $\operatorname{Coker}(L)$ as $y: \rightarrow(1 / \omega) \int_{0}^{\omega} y(t) d t$. It is obvious that

$$
\{u \mid u \in \operatorname{Ker} L \bigcap \partial \Omega\}=\left\{u \mid u \equiv N_{3} \text {, or }-N_{3}\right\} \text {. }
$$

Without loss of generality, suppose that $u \equiv N_{3}$; then,

$$
\begin{aligned}
{[Q N(u)+Q r, u][Q N(-u)+Q r, u] } \\
=N_{3}^{2}\left[\int_{0}^{\omega} r(t) d t\right. \\
\left.\quad-N_{3} \int_{0}^{\omega}\left(A(t)+\sum_{i=1}^{n} B_{i}(t)+\sum_{j=1}^{m} C_{j}(t)\right) d t\right]
\end{aligned}
$$

$$
\begin{aligned}
\times & {\left[\int_{0}^{\omega} r(t) d t\right.} \\
& \left.\quad+N_{3} \int_{0}^{\omega}\left(A(t)+\sum_{i=1}^{n} B_{i}(t)+\sum_{j=1}^{m} C_{j}(t)\right) d t\right] \\
= & \omega^{2} N_{3}^{2}\left[\bar{r}-N_{3}\left(\bar{A}+\sum_{i=1}^{n} \overline{B_{i}}+\sum_{j=1}^{m} \overline{C_{j}}\right)\right] \\
\times & {\left[\bar{r}+N_{3}\left(\bar{A}+\sum_{i=1}^{n} \overline{B_{i}}+\sum_{j=1}^{m} \overline{C_{j}}\right)\right], }
\end{aligned}
$$

since $N_{3}>\left|\bar{r} /\left(\bar{A}+\sum_{i=1}^{n} \overline{B_{i}}+\sum_{j=1}^{m} \overline{C_{j}}\right)\right|$, then

$$
\begin{gathered}
N_{3}>\frac{\bar{r}}{\bar{A}+\sum_{i=1}^{n} \overline{B_{i}}+\sum_{j=1}^{m} \overline{C_{j}}}, \\
-N_{3}<-\frac{\bar{r}}{\bar{A}+\sum_{i=1}^{n} \overline{B_{i}}+\sum_{j=1}^{m} \overline{C_{j}}} .
\end{gathered}
$$

By (46), we get that

$$
[Q N(u)+Q r, u][Q N(-u)+Q r, u]<0 .
$$

Therefore, by using Lemma 4, we obtain that (1) has at least one positive $\omega$-periodic solution; the proof of Theorem 13 is completed.

Since $\left|1-\tau_{l}^{\prime}\right|_{0}<1$, for all $l=1,2, \ldots, p$, it follows that $\sum_{l=1}^{p}\left|1-\tau_{l}^{\prime}\right|_{0}\left|D_{l}\right|_{0} \leq \sum_{l=1}^{p}\left|1-\tau_{l}^{\prime}\right|_{0}^{1 / 2}\left|D_{l}\right|_{0}<1$. So from Theorem 13, we have the following result.

Corollary 14. Suppose that $\left(H_{1}\right)-\left(H_{3}\right)$ and the following conditions hold:

$\left(H_{4}^{\prime}\right)$ there exists a constant $\eta>0$ such that $|\Gamma(t)| \geq \eta$, for all $t \in[0, \omega]$, where $\Gamma(t)$ is defined by $(23)$;

$$
\begin{gathered}
\left(H_{5}^{\prime}\right) \text { if }(1 / 2)\left(|A|_{0} \omega+\sum_{i=1}^{n}|B|_{0} \omega+\sum_{j=1}^{m}\left|C_{j}\right|_{0} \omega^{1 / 2}\right)+ \\
\sum_{l=1}^{p}\left|1-\tau_{l}^{\prime}\right|_{0}^{1 / 2}\left|D_{l}\right|_{0}<1 .
\end{gathered}
$$

Then (1) has at least one positive $\omega$-periodic solution.

On the other hand, if $\left|1-\tau_{l}^{\prime}\right|_{0}>1$, for all $l=1,2, \ldots, p$, it follows that $\sum_{l=1}^{p}\left|1-\tau_{l}^{\prime}\right|_{0}\left|D_{l}\right|_{0} \geq \sum_{l=1}^{p}\left|1-\tau_{l}^{\prime}\right|_{0}^{1 / 2}\left|D_{l}\right|_{0}$. So from Theorem 13, we also have the following result.

Corollary 15. Suppose that $\left(H_{1}\right)-\left(H_{3}\right)$ and the following conditions hold:

$\left(H_{4}^{*}\right)$ there exists a constant $\eta>0$ such that $|\Gamma(t)| \geq \eta$, for all $t \in[0, \omega]$, where $\Gamma(t)$ is defined by $(23)$;

$\left(H_{5}^{*}\right)$ if $(1 / 2)\left(|A|_{0} \omega+\sum_{i=1}^{n}|B|_{0} \omega+\sum_{j=1}^{m}\left|C_{j}\right|_{0} \omega^{1 / 2}\right)+$ $\sum_{l=1}^{p}\left|1-\tau_{l}^{\prime}\right|_{0}\left|D_{l}\right|_{0}<1$

Then, (1) has at least one positive w-periodic solution. 
Our next theorem is concerned with the global attractivity of periodic solution of the system (1).

Theorem 16. Suppose that $\left(H_{1}\right)-\left(H_{5}\right)$ and the following conditions hold:

$\left(H_{6}\right)$ there is a positive constant $\mu$ such that

$$
\begin{gathered}
\sum_{l=1}^{p}\left|D_{l}\right|_{0}+\int_{0}^{t}\left[\sum_{l=1}^{p}\left|H_{l}\right|_{0}+\sum_{i=1}^{n}\left|B_{i}\right|_{0}+\sum_{j=1}^{m}\left|C_{j}\right|_{0}\right] \\
\times \exp \left(-\int_{u}^{t} A(\xi) d \xi\right) d u \leq \mu<1 \\
\left(H_{7}\right) \exp \left(-\int_{0}^{t} A(\xi) d \xi\right) \rightarrow 0, \text { as } t \rightarrow+\infty
\end{gathered}
$$

Then, the positive $\omega$-periodic solution of (1) is globally attractive, where $H_{l}(t)=\left[D_{l}^{\prime}(t)+D_{l}(t) A(t)\right]\left[1-\tau_{l}^{\prime}(t)\right]+$ $D_{l}(t) \tau_{l}^{\prime \prime}(t) /\left[1-\tau_{l}^{\prime}(t)\right]^{2}$.

Proof. Suppose that $y(t)=e^{v(t)}$ is a positive $\omega$-periodic solution of (10), $y^{*}(t)=e^{v^{*}(t)}$ is another positive solution of (10). Similar to (17), we have

$$
\begin{aligned}
\frac{d v}{d t}=r( & t)-A(t) v(t)-\sum_{i=1}^{n} B_{i}(t) v\left(t-\sigma_{i}(t)\right) \\
& -\sum_{j=1}^{m} C_{j}(t) \int_{-\infty}^{t} K_{j}(t-s) v(s) d s \\
& -\sum_{l=1}^{p} D_{0 l}(t) v^{\prime}\left(t-\tau_{l}(t)\right), \\
\frac{d v^{*}}{d t}= & -(t)-A(t) v^{*}(t) \\
& -\sum_{i=1}^{n} B_{i}(t) v^{*}\left(t-\sigma_{i}(t)\right) \\
& -\sum_{j=1}^{m} C_{j}(t) \int_{-\infty}^{t} K_{j}(t-s) v^{*}(s) d s \\
& -\sum_{l=1}^{p} D_{0 l}(t) v^{\prime *}\left(t-\tau_{l}(t)\right) .
\end{aligned}
$$

Let $v(t)-v^{*}(t)=w(t)$; then,

$$
\begin{aligned}
\frac{d w}{d t}= & -A(t) w(t)-\sum_{i=1}^{n} B_{i}(t) w\left(t-\sigma_{i}(t)\right) \\
& -\sum_{j=1}^{m} C_{j}(t) \int_{-\infty}^{t} K_{j}(t-s) w(s) d s \\
& -\sum_{l=1}^{p} D_{0 l}(t) w^{\prime}\left(t-\tau_{l}(t)\right) .
\end{aligned}
$$

Multiply both sides of (51) with $\exp \left(\int_{0}^{t} A(\xi) d \xi\right)$ and then integrate from 0 to $t$ to obtain that

$$
\begin{gathered}
\int_{0}^{t}\left[w(u) \exp \left(\int_{0}^{u} A(\xi) d \xi\right)\right]^{\prime} d u \\
=-\int_{0}^{t}\left[\sum_{i=1}^{n} B_{i}(u) w\left(t-\sigma_{i}(u)\right)\right. \\
+\sum_{j=1}^{m} C_{j}(u) \int_{-\infty}^{t} K_{j}(u-s) w(s) d s \\
\left.+\sum_{l=1}^{p} D_{0 l}(u) w^{\prime}\left(u-\tau_{l}(u)\right)\right] \\
\times \exp \left(\int_{0}^{u} A(\xi) d \xi\right) d u,
\end{gathered}
$$

Then,

$$
\begin{aligned}
w(t)= & w(0) \exp \left(-\int_{0}^{t} A(\xi) d \xi\right) \\
-\int_{0}^{t}\left[\sum_{i=1}^{n} B_{i}(u) w\left(t-\sigma_{i}(u)\right)\right. & \\
& +\sum_{j=1}^{m} C_{j}(u) \int_{-\infty}^{t} K_{j}(u-s) w(s) d s \\
& \left.+\sum_{l=1}^{p} D_{0 l}(u) w^{\prime}\left(u-\tau_{l}(u)\right)\right] \\
& \times \exp \left(-\int_{u}^{t} A(\xi) d \xi\right) d u .
\end{aligned}
$$

Meanwhile, we see that

$$
\begin{gathered}
\int_{0}^{t} \sum_{l=1}^{p} D_{0 l}(u) w^{\prime}\left(u-\tau_{l}(u)\right) \exp \left(-\int_{u}^{t} A(\xi) d \xi\right) d u \\
=\int_{0}^{t} \sum_{l=1}^{p} \frac{D_{0 l}(u) w^{\prime}\left(u-\tau_{l}(u)\right)\left(1-\tau_{l}^{\prime}(u)\right)}{1-\tau_{l}^{\prime}(u)} \\
\quad \times \exp \left(-\int_{u}^{t} A(\xi) d \xi\right) d u \\
=\int_{0}^{t} \sum_{l=1}^{p}\left[\frac{D_{01}(u) \exp \left(-\int_{u}^{t} A(\xi) d \xi\right)}{1-\tau_{l}^{\prime}(u)}\right] \\
\times\left[w^{\prime}\left(u-\tau_{l}(u)\right)\left(1-\tau_{l}^{\prime}(u)\right)\right] d u
\end{gathered}
$$




$$
\begin{aligned}
& =\sum_{l=1}^{p}\left[\frac{D_{0 l}(t)}{1-\tau_{l}^{\prime}(t)} w\left(t-\tau_{l}(t)\right)\right. \\
& \left.\quad-\frac{D_{0 l}(0)}{1-\tau_{l}^{\prime}(0)} w\left(-\tau_{l}(0)\right) \exp \left(-\int_{0}^{t} A(\xi) d \xi\right)\right] \\
& -\sum_{l=1}^{p} \int_{0}^{t} H_{l}(u) \exp \left(-\int_{u}^{t} A(\xi) d \xi\right) w\left(u-\tau_{l}(u)\right) d u \\
& =\sum_{l=1}^{p}\left[D_{l}(t) w\left(t-\tau_{l}(t)\right)\right. \\
& \quad-D_{l=1}^{p} \int_{0}^{t} H_{l}(u) \exp \left(-\int_{u}^{t} A(\xi) d \xi\right) w\left(u-\tau_{l}(u)\right) d u,
\end{aligned}
$$

where

$$
\begin{aligned}
& H_{l}(u) \\
& =\frac{\left[D_{l}^{\prime}(u)+D_{l}(t) A(t)\right]\left[1-\tau_{l}^{\prime}(t)\right]+D_{l}(t) \tau_{l}^{\prime \prime}(t)}{\left[1-\tau_{l}^{\prime}(t)\right]^{2}} .
\end{aligned}
$$

Substituting (55) into (54), we get that

$$
\begin{aligned}
w(t)= & {\left[w(0)+\sum_{l=1}^{p} D_{l}(0) w\left(-\tau_{l}(0)\right)\right] } \\
& \times \exp \left(-\int_{0}^{t} A(\xi) d \xi\right) \\
& +\int_{0}^{t}\left[\sum_{l=1}^{p} H_{l}(u) w\left(u-\tau_{l}(u)\right)\right. \\
& \quad-\sum_{i=1}^{n} B_{i}(u) w\left(u-\sigma_{i}(u)\right) \\
& \left.\quad-\sum_{j=1}^{m} C_{j}(u) \int_{-\infty}^{u} K_{j}(u-s) w(s) d s\right] \\
& \times \exp \left(-\int_{u}^{t} A(\xi) d \xi\right) d u-\sum_{l=1}^{p} D_{l}(t) w\left(t-\tau_{l}(t)\right) .
\end{aligned}
$$

Therefore, we have

$$
\begin{aligned}
|w|_{0} \leq & \left|w(0)+\sum_{l=1}^{p} D_{l}(0) w\left(-\tau_{l}(0)\right)\right| \\
& \times \exp \left(-\int_{0}^{t} A(\xi) d \xi\right)
\end{aligned}
$$

$$
\begin{aligned}
& +\int_{0}^{t}\left[\sum_{l=1}^{p}\left|H_{l}\right|_{0}+\sum_{i=1}^{n}\left|B_{i}\right|_{0}+\sum_{j=1}^{m}\left|C_{j}\right|_{0}\right] \\
& \times|w|_{0} \exp \left(-\int_{u}^{t} A(\xi) d \xi\right) d u+\sum_{l=1}^{p}\left|D_{l}\right|_{0}|w|_{0} .
\end{aligned}
$$

From $\left(H_{6}\right)$, we have

$$
\begin{aligned}
& |w|_{0} \\
& \leq\left|w(0)+\sum_{l=1}^{p} D_{l}(0) w\left(-\tau_{l}(0)\right)\right| \exp \left(-\int_{0}^{t} A(\xi) d \xi\right) \\
& \quad \times\left(1-\sum_{l=1}^{p}\left|D_{l}\right|_{0}\right. \\
& \quad-\int_{0}^{t}\left[\sum_{l=1}^{p}\left|H_{l}\right|_{0}+\sum_{i=1}^{n}\left|B_{i}\right|_{0}+\sum_{j=1}^{m}\left|C_{j}\right|_{0}\right] \\
& \left.\times \exp \left(-\int_{u}^{t} A(\xi) d \xi\right) d u\right)^{-1} \\
& \leq \frac{\left|w(0)+\sum_{l=1}^{p} D_{l}(0) w\left(-\tau_{l}(0)\right)\right| \exp \left(-\int_{0}^{t} A(\xi) d \xi\right)}{1-\mu} .
\end{aligned}
$$

From $\left(H_{7}\right)$, we have

$$
|w|_{0}=\left|v-v^{*}\right|_{0}=0, \quad \text { as } t \longrightarrow+\infty,
$$

thus, $v(t) \rightarrow v^{*}(t)$, as $t \rightarrow+\infty$; that is, the positive $\omega$ periodic solution of (10) is globally attractive; by Definition 2, the positive $\omega$-periodic solution of (1) is globally attractive. The proof is completed.

Consider the following equation:

$$
\begin{aligned}
\frac{d N(t)}{d t}=N(t)[ & r(t)-a(t) \ln N(t) \\
& -\sum_{i=1}^{n} b_{i}(t) \ln N\left(t-\sigma_{i}(t)\right) \\
& -\sum_{j=1}^{m} c_{j}(t) \int_{-\infty}^{t} K_{j}(t-s) \ln N(s) d s \\
& \left.-\sum_{l=1}^{p} d_{l}(t) \frac{d \ln N\left(t-\tau_{l}(t)\right)}{d t}\right],
\end{aligned}
$$

which is a special case of the system (1) without impulse. Similarly, we can get the following conclusions. 
Corollary 17. Suppose that the following conditions hold:

$\left(H_{1}^{*}\right) r(t), a(t), b_{i}(t), c_{j}(t), d_{l}(t), \sigma_{i}(t), \tau_{l}(t)(i=1,2, \ldots$, $n ; j=1,2, \ldots, m ; l=1,2, \ldots, p)$ are all positive $\omega$-periodic continuous functions with $\sigma_{i}(t) \geq 0, \tau_{l}(t) \geq$ $0, t \in[0, \omega], \sigma_{i}^{\prime}(t)<1, \tau_{l}^{\prime}(t)<1$, for all $i=\{1,2, \ldots$, $n\}$, for all $l=\{1,2, \ldots, p\}$; furthermore, $d_{l}(t) \in$ $C^{1}(R, R), \tau_{l}(t) \in C^{2}(R, R), \int_{0}^{\infty} K_{j}(s) d s=1, \int_{0}^{+\infty} s K_{j}(s) d s$ $<+\infty$, for all $j=\{1,2, \ldots, m\}$, for all $l=\{1,2, \ldots$, $p\}$;

$\left(H_{2}^{*}\right)$ there exists a constant $\eta>0$ such that $\left|\Gamma^{*}(t)\right| \geq \eta$, for all $t \in[0, \omega]$, where $\Gamma^{*}(t)$ is defined by the following:

$$
\begin{aligned}
\Gamma^{*}(t):= & a(t)+\sum_{i=1}^{n} \frac{a_{i}\left(\zeta_{i}(t)\right)}{1-\sigma_{i}^{\prime}\left(\zeta_{i}(t)\right)} \\
& +\sum_{j=1}^{m} c_{j}(t)-\sum_{l=1}^{p} \frac{d_{l}^{\prime}\left(\xi_{l}(t)\right)}{1-\tau_{l}^{\prime}\left(\xi_{l}(t)\right)}
\end{aligned}
$$

$$
\begin{gathered}
\left(H_{3}^{*}\right) \text { if }(1 / 2)\left(|a|_{0} \omega+\sum_{i=1}^{n}\left|b_{i}\right|_{0} \omega+\sum_{j=1}^{m}\left|c_{j}\right|_{0} \omega^{1 / 2}\right)+\sum_{l=1}^{p} \mid 1- \\
\left.\tau_{l}^{\prime}\right|_{0} ^{1 / 2}\left|d_{l}\right|_{0}<1 \text {, and } \sum_{l=1}^{p}\left|1-\tau_{l}^{\prime}\right|_{0}\left|d_{l}\right|_{0}<1 .
\end{gathered}
$$

Then, (61) has at least one positive w-periodic solution.

Corollary 18. Suppose that $\left(H_{1}^{*}\right)$ and the following conditions hold:

$\left(H_{4}^{*}\right)$ there is a positive constant $\mu$ such that

$$
\begin{array}{r}
\sum_{l=1}^{p}\left|d_{l}\right|_{0}+\int_{0}^{t}\left[\sum_{l=1}^{p}\left|h_{l}\right|_{0}+\sum_{i=1}^{n}\left|b_{i}\right|_{0}+\sum_{j=1}^{m}\left|c_{j}\right|_{0}\right] \\
\times \exp \left(-\int_{u}^{t} a(\xi) d \xi\right) d u \leq \mu<1 ; \\
\left(H_{5}^{*}\right) \exp \left(-\int_{0}^{t} A(\xi) d \xi\right) \rightarrow 0 \text {, ast } \rightarrow+\infty .
\end{array}
$$

Then, the positive w-periodic solution of (61) is globally attractive, where $h_{l}(t):=\left[d_{l}^{\prime}(t)+d_{l}(t) a(t)\right]\left[1-\tau_{l}^{\prime}(t)\right]+$ $d_{l}(t) \tau_{l}^{\prime \prime}(t) /\left[1-\tau_{l}^{\prime}(t)\right]^{2}$.

Remark 19. One could easily see that the systems (5)-(7) are all special cases of the system (61); we can get the similar results, and we omit them here. Hence, our results improve and generalize the corresponding results in [4-7].

\section{An Example}

Consider the following impulsive model:

$$
\begin{aligned}
\frac{d N(t)}{d t}=N(t)[ & r(t)-a(t) \ln N(t) \\
& -b(t) \ln N(t-\sigma(t))
\end{aligned}
$$

$$
\begin{gathered}
-c(t) \int_{-\infty}^{t} K(t-s) \ln N(s) d s \\
\left.-d(t) \frac{d \ln N(t-\tau(t))}{d t}\right], \\
\Delta N\left(t_{k}\right)=N\left(t_{k}^{+}\right)-N\left(t_{k}\right) \\
=\theta_{k} N\left(t_{k}\right), \quad k=1,2, \ldots,
\end{gathered}
$$

where $r(t), a(t), b(t), c(t), d(t), \sigma(t), \tau(t)$ are all positive $\omega$ periodic continuous functions with $\sigma(t) \geq 0, \tau(t) \geq$ $0, t \in[0, \omega], \sigma^{\prime}(t)<1, \tau^{\prime}(t)<1$; furthermore, $d(t) \in$ $C^{1}(R, R), \tau(t) \in C^{2}(R, R), \int_{0}^{\infty} K(s) d s=1, \int_{0}^{+\infty} s K(s) d s<$ $+\infty$.

Corollary 20. Suppose that $\left(H_{1}\right)-\left(H_{3}\right)$ and the following conditions hold:

$\left(H_{4}\right)$ there exists a constant $\eta>0$ such that $|\Gamma(t)| \geq \eta$, for all $t \in[0, \omega]$, where $\Gamma(t)$ is defined by the following:

$$
\Gamma(t):=A(t)+\frac{B(\zeta(t))}{1-\sigma^{\prime}(\zeta(t))}+C(t)-\frac{D^{\prime}(\xi(t))}{1-\tau^{\prime}(\xi(t))} ;
$$

$\left(H_{5}\right)$ if $(1 / 2)\left[\left(|A|_{0}+|B|_{0}\right) \omega+|C|_{0} \omega^{1 / 2}\right)+\left|1-\tau^{\prime}\right|_{0}^{1 / 2}|D|_{0}<1$, and $\left|1-\tau^{\prime}\right|_{0}|D|_{0}<1$.

Then, (64) has at least one positive w-periodic solution, where

$$
\begin{gathered}
A(t)=a(t) \prod_{0<t_{k}<t}\left(1+\theta_{k}\right), \\
B(t)=b(t) \prod_{0<t_{k}<t-\sigma(t)}\left(1+\theta_{k}\right), \\
C(t)=c(t) \prod_{0<t_{k}<t}\left(1+\theta_{k}\right), \\
D(t)=d(t) \prod_{0<t_{k}<t-\tau(t)}\left(1+\theta_{k}\right) .
\end{gathered}
$$

Corollary 21. Suppose that $\left(H_{1}\right)-\left(H_{5}\right)$ and the following conditions hold:

$\left(H_{6}\right)$ there is a positive constant $\mu$ such that

$$
\begin{gathered}
|D|_{0}+\int_{0}^{t}\left[|H|_{0}+|B|_{0}+|C|_{0}\right] \\
\quad \times \exp \left(-\int_{u}^{t} A(\xi) d \xi\right) d u \leq \mu<1 \\
\left(H_{7}\right) \exp \left(-\int_{0}^{t} A(\xi) d \xi\right) \rightarrow 0, \text { as } t \rightarrow+\infty
\end{gathered}
$$

Then, the positive $\omega$-periodic solution of (64) is globally attractive, where $H(t):=\left[D^{\prime}(t)+D(t) A(t)\right]\left[1-\tau^{\prime}(t)\right]+$ $D(t) \tau^{\prime \prime}(t) /\left[1-\tau^{\prime}(t)\right]^{2}$.

Remark 22. The results in the work show that by means of appropriate impulsive perturbations, we can control the dynamics of these equations. 


\section{Acknowledgments}

This work was supported by the Construct Program of the Key Discipline in Hunan Province. Research was supported by the National Natural Science Foundation of China (10971229, 11161015), the China Postdoctoral Science Foundation (2012M512162), and Hunan Provincial Natural ScienceHengyang United Foundation of China (11JJ9002).

\section{References}

[1] K. Gopalsamy, Stability and Oscillation in Delay Differential Equations of Population Dynamics, vol. 74 of Mathematics and Its Applications, Kluwer Academic, Dordrecht, The Netherlands, 1992.

[2] G. Kirlinger, "Permanence in Lotka-Volterra equations: linked prey-predator systems," Mathematical Biosciences, vol. 82, no. 2, pp. 165-191, 1986.

[3] Y. K. Li, "Attractivity of a positive periodic solution for all other positive solution in a delay population model," Applied Mathematics, vol. 12, no. 3, pp. 279-282, 1997 (Chinese).

[4] Y. K. Li, "On a periodic neutral delay logarithmic population model," Journal of Systems Science and Complexity, vol. 19, no. 1, pp. 34-38, 1999 (Chinese).

[5] S. P. Lu and W. G. Ge, "Existence of positive periodic solutions for neutral logarithmic population model with multiple delays," Journal of Computational and Applied Mathematics, vol. 166, no. 2, pp. 371-383, 2004.

[6] Y. Luo and Z. G. Luo, "Existence of positive periodic solutions for neutral multi-delay logarithmic population model," Applied Mathematics and Computation, vol. 216, no. 4, pp. 1310-1315, 2010.

[7] Q. Wang, Y. Wang, and B. X. Dai, "Existence and uniqueness of positive periodic solutions for a neutral Logarithmic population model," Applied Mathematics and Computation, vol. 213, no. 1, pp. 137-147, 2009.

[8] F. Hui and L. Jibin, "On the existence of periodic solutions of a neutral delay model of single-species population growth," Journal of Mathematical Analysis and Applications, vol. 259, no. 1, pp. 8-17, 2001.

[9] S. P. Lu and W. G. Ge, "Existence of positive periodic solutions for neutral population model with multiple delays," Applied Mathematics and Computation, vol. 153, no. 3, pp. 885-902, 2004.

[10] Z. H. Yang and J. D. Cao, "Positive periodic solutions of neutral Lotka-Volterra system with periodic delays," Applied Mathematics and Computation, vol. 149, no. 3, pp. 661-687, 2004.

[11] H. F. Huo, "Existence of positive periodic solutions of a neutral delay Lotka-Volterra system with impulses," Computers and Mathematics with Applications, vol. 48, no. 12, pp. 1833-1846, 2004.

[12] F. D. Chen, "On a nonlinear nonautonomous predator-prey model with diffusion and distributed delay," Journal of Computational and Applied Mathematics, vol. 180, no. 1, pp. 33-49, 2005.

[13] J. R. Yan and A. M. Zhao, "Oscillation and stability of linear impulsive delay differential equations," Journal of Mathematical Analysis and Applications, vol. 227, no. 1, pp. 187-194, 1998.

[14] J. S. Yu, "Explicit conditions for stability of nonlinear scalar delay differential equations with impulses," Nonlinear Analysis: Theory, Methods and Applications, vol. 46, no. 1, pp. 53-67, 2001.
[15] Y. H. Xia, "Positive periodic solutions for a neutral impulsive delayed Lotka-Volterra competition system with the effect of toxic substance," Nonlinear Analysis: Real World Applications, vol. 8, no. 1, pp. 204-221, 2007.

[16] Q. Wang and B. X. Dai, "Existence of positive periodic solutions for a neutral population model with delays and impulse," Nonlinear Analysis: Theory, Methods and Applications, vol. 69, no. 11, pp. 3919-3930, 2008.

[17] Y. Zhang and J. Sun, "Stability of impulsive functional differential equations," Nonlinear Analysis: Theory, Methods and Applications, vol. 68, no. 12, pp. 3665-3678, 2008.

[18] G. P. Pang, F. Y. Wang, and L. S. Chen, "Extinction and permanence in delayed stage-structure predator-prey system with impulsive effects," Chaos, Solitons and Fractals, vol. 39, no. 5, pp. 2216-2224, 2009.

[19] J. O. Alzabut, "Almost periodic solutions for an impulsive delay Nicholson's blowflies model," Journal of Computational and Applied Mathematics, vol. 234, no. 1, pp. 233-239, 2010.

[20] M. Benchohra, J. Henderson, and S. K. Ntouyas, Impulsive Differential Equations and Inclusions, vol. 2, Hindawi Publishing Corporation, New York, NY, USA, 2006.

[21] D. D. Bainov and P. Simeonov, Impulsive Differential Equations: Periodic Solutions and Applications, Longman, Essex, UK, 1993.

[22] A. M. Samoikleno and N. A. Perestyuk, Impulsive Differential Equations, World Scientific, Singapore, 1995.

[23] D. J. Guo, Nonlinear Functional Analysis, Shandong Science and Technology Press, 2001.

[24] W. V. Petryshyn and Z. S. Yu, "Existence theorems for higher order nonlinear periodic boundary value problems," Nonlinear Analysis, vol. 6, no. 9, pp. 943-969, 1982.

[25] Z. D. Liu and Y. P. Mao, "Existence theorem for periodic solutions of higher order nonlinear differential equations," Journal of Mathematical Analysis and Applications, vol. 216, no. 2, pp. 481-490, 1997.

[26] Y. G. Zhou and X. H. Tang, "On existence of periodic solutions of Rayleigh equation of retarded type," Journal of Computational and Applied Mathematics, vol. 203, no. 1, pp. 1-5, 2007.

[27] Q. Wang, B. X. Dai, and Y. M. Chen, "Multiple periodic solutions of an impulsive predator-prey model with Hollingtype IV functional response," Mathematical and Computer Modelling, vol. 49, no. 9-10, pp. 1829-1836, 2009. 


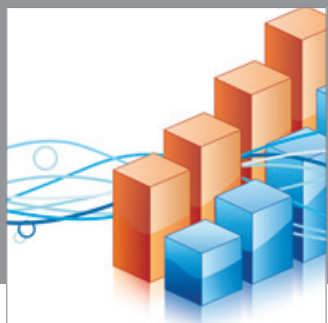

Advances in

Operations Research

mansans

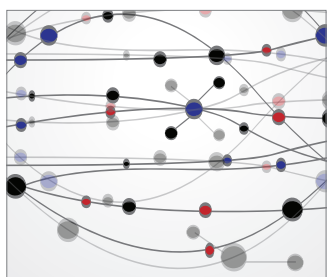

The Scientific World Journal
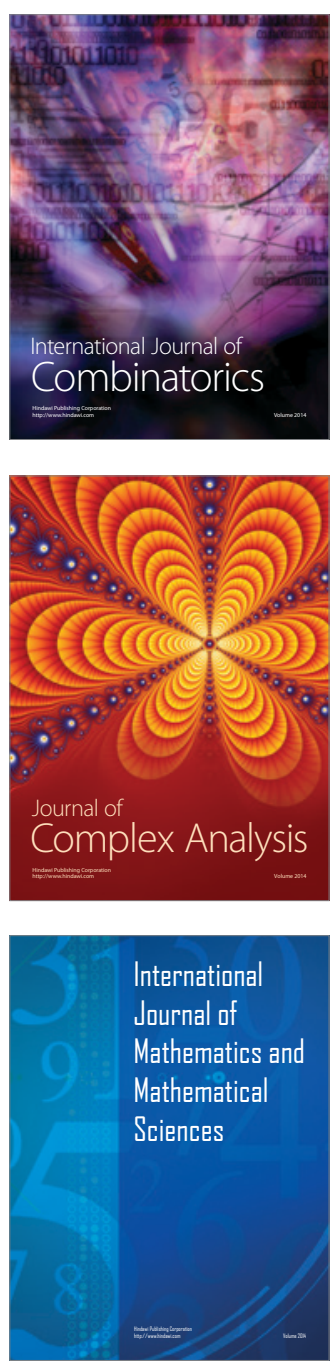
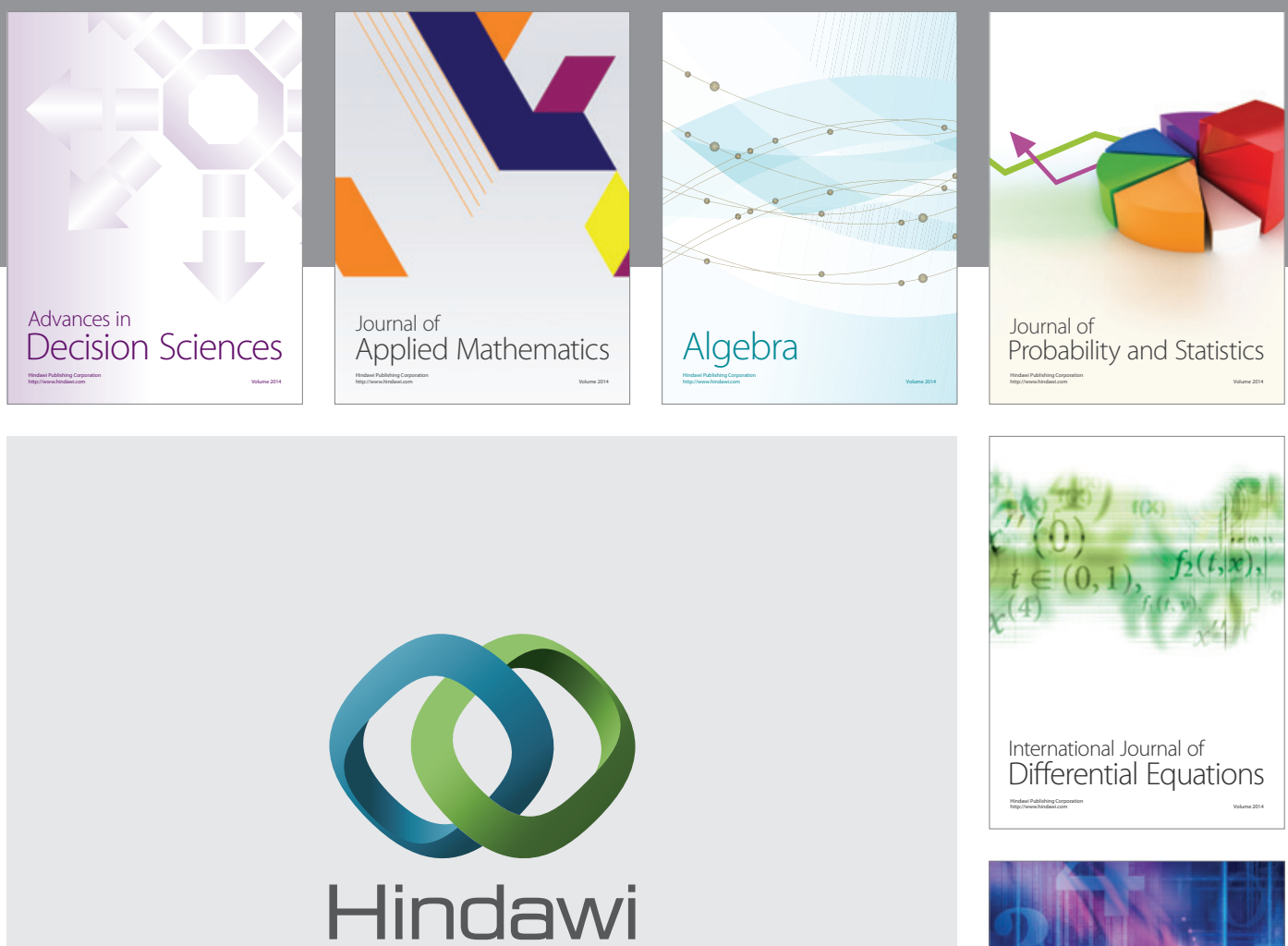

Submit your manuscripts at http://www.hindawi.com
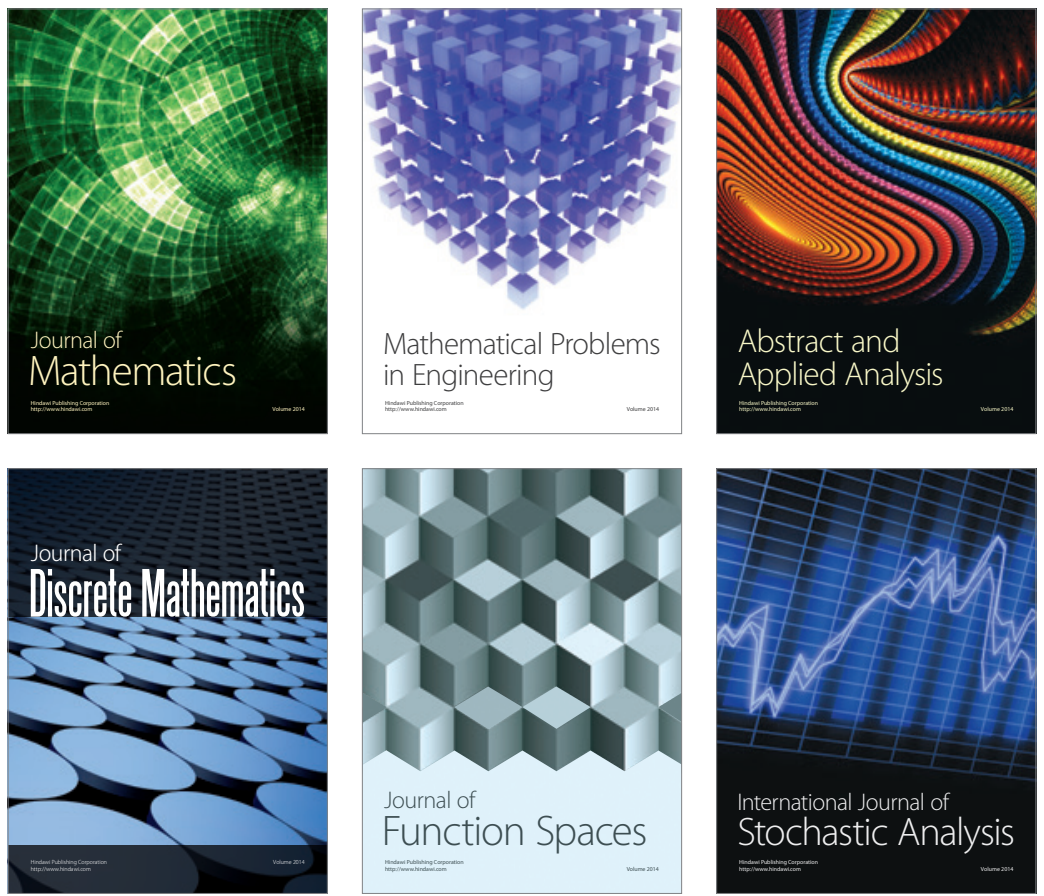

Journal of

Function Spaces

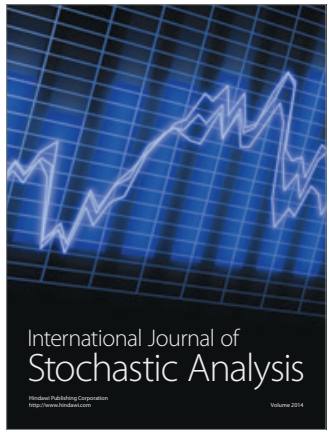

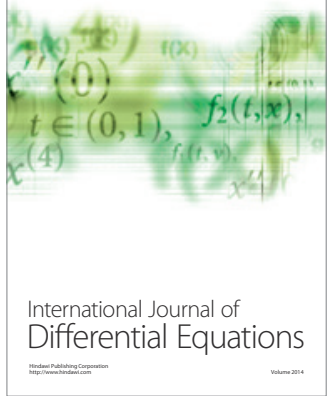
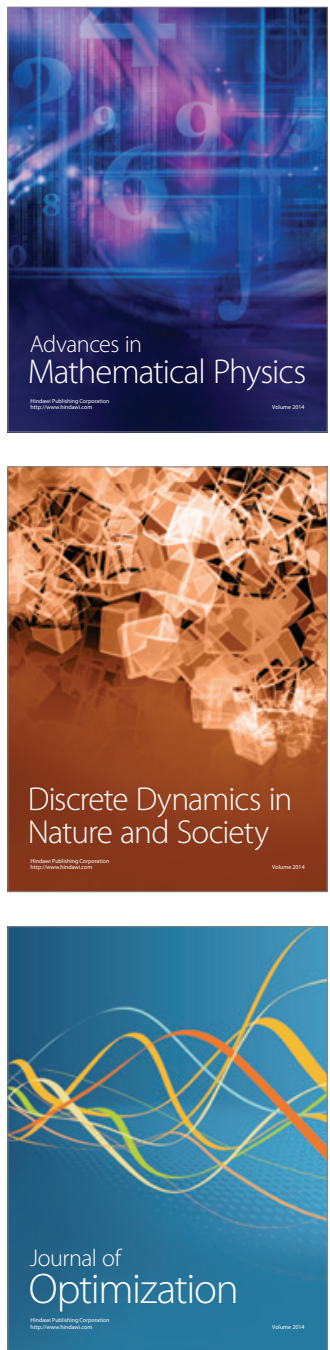